\title{
ON AN EXTREMAL PROBLEM IN FOURIER SERIES
}

\section{Lee Lorch}

\author{
(received April 1, 1960)
}

Let $f(x)$ be a bounded odd function, $-\pi<x<\pi,|f(x)| \leqslant 1$, with non-negative Fourier coefficients $b_{k}, k=1,2, \ldots$.

Otto Szász [1] proved anew the existence of a bounded set of numbers $\left\{\beta_{n}\right\}, n=1,2, \ldots$, such that

$$
\sum_{k=1}^{n} k b_{k} \leqslant \beta_{n} n \text {, }
$$

where $\beta_{\mathrm{n}}$ is the smallest constant satisfying the above inequality and added that $2 / \pi \leqslant \beta_{n} \leqslant 4 / \pi$. He pointed out [1, p. 170] that $\beta_{1}=4 / \pi$ and raised the question of the value of $\beta_{n}$ for $\mathrm{n}>1$.

The purpose of this note is to prove that $\beta_{n}=4 / \pi$ for each $n, n=1,2, \ldots$.

Proof. For each given $n$, the function $f(x)=\operatorname{sgn}\{\sin n x\}$ satisfies the requirements placed on $f(x)$, where, as usual, $\operatorname{sgn} A$ is 1 for $A$ positive, 0 for $A$ zero, -1 for A negative.

For this function we have

$$
\operatorname{sgn}\{\sin n x\}=\frac{4}{\pi} \sum_{k=0}^{\infty} \frac{\sin (2 k+1) n x}{2 k+1},
$$

so that $b_{n}=4 / \pi$ and $b_{k}=0$ for $k=0,1, \ldots, n-1$. Thus

$$
\sum_{k=1}^{n} k b_{k}=(4 / \pi) n
$$

and the proof is complete, since Szász showed that $\beta_{n} \leqslant 4 / \pi$.

\section{REFERENCE}

1. Otto Szász, Some extremum problems in the theory of Fourier series, Amer. J. of Math. 61 (1939), 165-177.

University of Alberta 